\title{
CPT/Lorentz Invariance Violation and Quantum Field Theory
}

\author{
Jorge Gamboa* \\ Departamento de Física, Universidad de Santiago de Chile \\ Casilla 307, Santiago 2, Chile \\ E-mail: jgamboa@lauca.usach.cl

\section{Paola Arias} \\ Departamento de Física, Universidad de Santiago de Chile \\ Casilla 307, Santiago 2, Chile \\ E-mail: paola.ariasegmail.com
}

\section{Ashok Das}

Department of Physics and Astronomy, University of Rochester, Rochester, NY 14627-0171, USA

and

Saha Institute of Nuclear Physics, 1/AF Bidhannagar, Calcutta 700064, India.

E-mail: das@pas.rochester.edu

\section{Justo Lopez-Sarrion}

Department of Physics, City College of CUNY

New York, NY 10031, USA

E-mail: justinux75@gmail.com

\section{Fernando Mendez}

Departamento de Física, Universidad de Santiago de Chile

Casilla 307, Santiago 2, Chile

E-mail: fmendez@lauca.usach.cl

\begin{abstract}
Analogies between the noncommutative harmonic oscillator and noncommutative fields are analyzed. Following this analogy we construct examples of quantum fields theories with explicit CPT and Lorentz symmetry breaking. Some applications to baryogenesis and neutrino oscillation are also discussed.
\end{abstract}

Fifth International Conference on Mathematical Methods in Physics - IC2006 April 24-28 2006

Centro Brasilerio de Pesquisas Fisicas, Rio de Janeiro, Brazil

\footnotetext{
*Speaker.
} 


\section{Introduction}

The Lorentz and CPT symmetries are fundamental cornerstones in the twentieth century for which there are not clear indications if persists at very high energies. By other hand, from effective theories point of view, it seems natural to think that the presently quantum field theories (QFT) and the symmetry principles they are based on, remain valid just for a given range of energies beyond which, possibly new and unexpected phenomena could emerge. For example, the relativistic invariance itself could be broken or deformed [1].

If QFT describes fundamental interactions for any energy range, then it seems natural to think that any QFT -seen as effective theory- must incorporate two energy scales, namely, the infrared and ultraviolet ones. Both scales might give rise to non conventional implications.

There are at least two examples of the previous idea, where the infrared scale can be very important. The first one, is the physics involved in the infrared sector of QED where still several technical aspects need to be understood as well as many conceptual problems still remain open [2].

The infrared sector of QED is the natural link between quantum field theory and quantum mechanics and then we ask which are the problems and how can we understand the physics in this interface?, what are the most convenient approximation criteria? In spite of many efforts performed during the fifties and sixties this problem still have not been clarified.

In the same context, another important example is QCD where the physical picture in the infrared limit is nontrivial because, at low energy, the theory is nonperturbative and phenomena such as confinement or hadronization should be solved using new methods beyond perturbation theory.

In this paper we would like to report our previous results on the idea of how the Lorentz symmetry could be broken in a QFT and also to point out new progress in the application of this to neutrino physics.

In order to expose our results, we will start in section II describing noncommutative mechanics and the harmonic oscillator in order to introduce, in the same section, the notion of noncommutative scalar and gague fields. In section III we we will report our recent progress in neutrino physics when noncommutative fermion fields are introduced. Section IV is devoted to resume the results of previous ideas when they are applied to the study of early universe physics. In the last section, conclusion and outlook is presented.

\section{Noncommutative quantum mechanics and noncommutative fields}

In this section we will study the non commutative quantum harmonic oscillator and how it can be used to define a non commutative field theory. As a warm up exercise let us consider first the standard bidimensional quantum harmonic oscillator described by the following Hamiltonian operator

$$
H=\frac{\omega}{2}\left[p_{1}^{2}+p_{2}^{2}+q_{1}^{2}+q_{2}^{2}\right],
$$

with standard commutation relations $(i, j=1,2)$

$$
\begin{aligned}
{\left[q_{i}, q_{j}\right] } & =\left[p_{i}, p_{j}\right]=0, \\
{\left[q_{i}, p_{j}\right] } & =i \delta_{i j} .
\end{aligned}
$$


Note that variables $\left\{q_{i}, p_{j}\right\}$ are dimensionless and are related with usual ones $\left\{Q_{i}, P_{j}\right\}$ by $q_{i}=$ $\sqrt{m \omega} Q_{i}$ and $p_{i}=(m \omega)^{-1 / 2} P_{i}$.

The dynamics of this system is described by the Heisenberg equations

$$
i \frac{d \mathscr{O}}{d t}=[\mathscr{O}, H]
$$

which, specified to (2.1) and (2.2) give rise to

$$
\begin{aligned}
\dot{q}_{i} & =\omega p_{i}, \\
\dot{p}_{i} & =-\omega q_{i} .
\end{aligned}
$$

The system (2.4) is equivalent to the very well known second order differential equation

$$
\ddot{q}_{i}=-\omega^{2} q_{i} .
$$

Therefore, the solution of (2.4) turn out to be

$$
\begin{aligned}
& q_{i}(t)=A_{i} e^{i \omega t}+B_{i} e^{-i \omega t}, \\
& p_{i}(t)=i A_{i} e^{i \omega t}-i B_{i} e^{-i \omega t} .
\end{aligned}
$$

The algebra of operators $A_{i}$ and $B_{i}$ can be fixed using the canonical algebra (2.2). Indeed, replacing (2.6) in (2.2) we find that

$$
\begin{aligned}
& {\left[A_{i}, A_{j}\right]=\left[B_{i}, B_{j}\right]=0,} \\
& {\left[A_{i}, B_{j}\right]=-\frac{1}{2} \delta_{i j} .}
\end{aligned}
$$

Then we can identify

$$
\sqrt{2} A_{i} \rightarrow a_{i}^{\dagger}, \quad \sqrt{2} B_{i} \rightarrow a_{i}
$$

and the algebra (2.7) becomes

$$
\begin{aligned}
{\left[a_{i}, a_{j}\right] } & =\left[a_{i}^{\dagger}, a_{j}^{\dagger}\right]=0, \\
{\left[a_{i}, a_{j}^{\dagger}\right] } & =\delta_{i j} .
\end{aligned}
$$

In terms of $a_{i}^{\dagger}$ and $a_{i}$, as is well known, one find that the Hamiltonian becomes

$$
H=\omega\left(a_{1}^{\dagger} a_{1}+a_{2}^{\dagger} a_{2}+1\right)
$$

The construction of the Hilbert space is straightforward.

Using these results let us solve the noncommutative harmonic oscillator described by the Hamiltonian (2.1) but commutation relations deformed as follows:

$$
\left[q_{i}, q_{j}\right]=i \theta \varepsilon_{i j}, \quad\left[p_{i}, p_{j}\right]=i \mathscr{B}, \varepsilon_{i j} \quad\left[q_{i}, p_{j}\right]=i \delta_{i j}
$$

where $\theta$ and $\mathscr{B}$ are parameters "measuring" noncommutativity in $q$ and $p$ respectively ${ }^{1}$.

\footnotetext{
${ }^{1}$ Note that this parameters are dimensionless in our notation, but they actually have dimensions in the standard variables.
} 
Using the Hamiltonian (2.1) and (2.11) one find that equations of motion are

$$
\begin{aligned}
& \dot{q}_{i}=\omega\left(p_{i}+\theta \varepsilon_{i j} q_{j}\right), \\
& \dot{p}_{i}=\omega\left(-q_{i}+\mathscr{B} \varepsilon_{i j} p_{j}\right) .
\end{aligned}
$$

Following the same procedure previously discussed, one find that the analogous to (2.5) turn out to be now

$$
\ddot{q}_{i}=\omega(\theta+\mathscr{B}) \varepsilon_{i j} \dot{q}_{j}+\omega^{2}(\mathscr{B} \theta-1) q_{i} .
$$

Although this set of equations are coupled ones, one decouple these equations using the following trick: let us define the complex variable $Z=q_{1}+i q_{2}$, then (2.13) can be written as

$$
\ddot{Z}=-i \omega(\theta+\mathscr{B}) \dot{Z}+\omega^{2}(\mathscr{B} \theta-1) Z,
$$

and a similar equation for the conjugate $Z^{\dagger}$. This problem is formally is equivalent to the damped harmonic oscillator.

As in the standard case, we look now for a solution with the shape

$$
Z(t)=e^{\alpha t}
$$

which imply that

$$
\alpha^{2}+i \omega(\theta+\mathscr{B}) \alpha-\omega^{2}(\mathscr{B} \theta-1)=0,
$$

and as a consequence the possible values of $\alpha$ are

$$
\frac{\alpha_{ \pm}}{\omega}=i\left(-\frac{\theta+\mathscr{B}}{2} \pm \sqrt{1+\left(\frac{\theta-\mathscr{B}}{2}\right)^{2}}\right) .
$$

Thus, the general solution of the noncommutative harmonic oscillator is

$$
Z(t)=A_{+} e^{i \alpha_{+} t}+A_{-} e^{i \alpha_{-} t},
$$

where $A_{ \pm}$are complex operators. Note that there is a redefinition of $\alpha_{ \pm}$since we have factorized the $i$ in (2.15). It is interesting to note also that the solution (2.16) is a superposition of two oscillation modes, one positive (because $\alpha_{+}>0$ ) and other negative (because $\alpha_{-}<0$ ). Therefore, the solution has the same structure of the standard case, but there is an asymmetry due to the fact that $\left|\alpha_{-}\right| \neq \alpha_{+}$.

Of course from (2.16) it is possible to compute $q_{i}(t)$, and from the equation of motion we obtain $p_{i}$

$$
\begin{aligned}
& q_{j}=\frac{(-i)^{j-1}}{2}\left[a+b-(-)^{j}\left(a^{\dagger}+b^{\dagger}\right)\right], \\
& p_{j}=-\frac{(-i)^{j}}{2}\left[\lambda_{+}\left(a+(-)^{j} a^{\dagger}\right)+\lambda_{-}\left(b+(-)^{j} b^{\dagger}\right)\right] .
\end{aligned}
$$

with $a=A_{+} e^{i \alpha_{+} t}, b=A_{-} e^{i \alpha_{-} t}$ and $\lambda_{ \pm}=\theta+\alpha_{ \pm} / \omega$

Following the example of the commutative case, we must find the commutation relation between operators $A_{ \pm}$and $A_{ \pm}^{\dagger}$ from the known relations (2.11). 
Since the result of this commutators do not depend on time, the following condition fulfills

$$
\left[A_{+}, A_{-}\right]=0=\left[A_{+}, A_{-}^{\dagger}\right] .
$$

Note that the remaining commutators, $\left[A_{ \pm}, A_{ \pm}^{\dagger}\right]$ are obtained from the conditions $\left[q_{1}, q_{2}\right]=i \theta$ and $\left[p_{1}, p_{2}\right]=i B$ and then, the condition $\left[q_{1}, p_{1}\right]=i$ is just a consistency check. After a straightforward calculation we obtain

$$
\left[A_{ \pm}, A_{ \pm}^{\dagger}\right]=\mp 2 \frac{1+\theta \lambda_{\mp}}{\lambda_{+}-\lambda_{-}} .
$$

This equation shows that non commutative harmonic oscillator in two dimensions is equivalent to two one-dimensional harmonic oscillator.

In fact, by a rescaling of $A_{ \pm}$operators

$$
\tilde{A}_{+}=\left(\frac{\lambda_{+}-\lambda_{-}}{2\left(1+\theta \lambda_{-}\right)}\right)^{1 / 2} A_{+}^{\dagger}, \quad \tilde{A}_{-}=\left(\frac{\lambda_{+}-\lambda_{-}}{2\left(1+\theta \lambda_{+}\right)}\right)^{1 / 2} A_{-},
$$

and similar relations for $\tilde{A_{ \pm}^{\dagger}}$, we find

$$
\left[\tilde{A}_{+}, \tilde{A}_{+}^{\dagger}\right]=1=\left[\tilde{A}_{-}, \tilde{A}_{-}^{\dagger}\right] .
$$

Operators $\tilde{A}_{ \pm}$play the role of standard lowering and rising operator as in the commutative case. The Hamiltonian of this two dimensional non commutative harmonic oscillator is

$$
H=\omega_{+}\left(\tilde{A}_{+} \tilde{A}_{+}^{\dagger}+1 / 2\right)+\omega_{-}\left(\tilde{A}_{-} \tilde{A}_{-}^{\dagger}+1 / 2\right) .
$$

with $\omega_{ \pm}=\sqrt{1+\left(\frac{\theta-B}{2}\right)^{2}} \pm\left(\frac{\theta+B}{2}\right)$.

Previous result is the starting point for constructing noncommutative complex scalar field theory [3]. Consider the standard relativistic Hamiltonian density for a complex scalar field

$$
\mathscr{H}=\Pi^{\dagger} \Pi+\nabla \Phi^{\dagger} \nabla \Phi+m^{2} \Phi^{\dagger} \Phi
$$

plus non standard commutation relations

$$
\begin{aligned}
{\left[\Phi(\mathbf{x}), \Phi^{\dagger}\left(\mathbf{x}^{\prime}\right)\right] } & =\theta \delta^{3}\left(\mathbf{x}-\mathbf{x}^{\prime}\right), \\
{\left[\Pi(\mathbf{x}), \Pi^{\dagger}\left(\mathbf{x}^{\prime}\right)\right] } & =B \delta^{3}\left(\mathbf{x}-\mathbf{x}^{\prime}\right), \\
{\left[\Phi(\mathbf{x}), \Pi\left(\mathbf{x}^{\prime}\right)\right] } & =\delta^{3}\left(\mathbf{x}-\mathbf{x}^{\prime}\right),
\end{aligned}
$$

where $\theta$ and $B$ parameterizes the non commutativity in the field space and have dimensions of energy $^{-1}$ and energy, respectively.

In the standard case $(\theta=0=B)$, quantized fields are a superposition of quantum harmonic oscillators with frequency $\omega(\mathbf{p})=\sqrt{\mathbf{p}^{2}+m^{2}}$, one for each value of momenta $\mathbf{p}$. The structure of these linear superpositions are given by (2.6).

For non commutative fields, the constructions proceeds in a similar way. The analog of (2.6) is given by (2.17) once they are expressed in terms of operators $\tilde{A}_{ \pm}$. Therefore we consider now a linear superposition with

$$
\begin{aligned}
& \Phi(\mathbf{x})=\int \frac{d^{3} p}{(2 \pi)^{3}} \frac{1}{\sqrt{\omega}}\left[\eta \varepsilon_{1} a_{\mathbf{p}} e^{i \mathbf{p x}}+\varepsilon_{2} b_{\mathbf{p}}^{\dagger} e^{-i \mathbf{p x}}\right], \\
& \Pi(\mathbf{x})=i \int \frac{d^{3} p}{(2 \pi)^{3}} \sqrt{\omega}\left[-\varepsilon_{1} a_{\mathbf{p}} e^{i \mathbf{p x}}+\eta \varepsilon_{2} b_{\mathbf{p}}^{\dagger} e^{-i \mathbf{p x}}\right],
\end{aligned}
$$


where coefficients $\eta$ and $\varepsilon_{i}$ are those appearing in the previously mentioned linear superposition, but with $\omega$ replaced by $\omega(\mathbf{p})$. We are following notation of [3] where $\eta=\lambda_{+}$and $\varepsilon_{1}^{2}=\left(\lambda_{+}+\right.$ $B)\left(\lambda_{+}^{2}+1\right)^{-1}$ and $\varepsilon_{1}^{2}=\left(\lambda_{+}-\theta\right)\left(\lambda_{+}^{2}+1\right)^{-1}$. Note that dependences on $\mathbf{p}$ are inherited from $\omega(\mathbf{p})$.

Operators $a, b$, by other hand, satisfy the canonical algebra

$$
\left[a_{\mathbf{p}}, a_{\mathbf{p}^{\prime}}^{\dagger}\right]=(2 \pi)^{3} \delta^{3}\left(\mathbf{p}-\mathbf{p}^{\prime}\right), \quad\left[b_{\mathbf{p}}, b_{\mathbf{p}^{\prime}}^{\dagger}\right]=(2 \pi)^{3} \delta^{3}\left(\mathbf{p}-\mathbf{p}^{\prime}\right),
$$

and are in correspondence with $\tilde{A}_{ \pm}$. Is straightforward to prove that fields constructed in this way satisfy the commutation relations (2.21).

The Hamiltonian of this theory

$$
H=\int d^{3} x \mathscr{H}(x)
$$

with the density (2.20) expressed in terms of the non commutative fields (2.22) is a superposition of two anisotropic oscillators (2.19) where frequencies are now $\omega(\mathbf{p})$. That is

$$
H=\int \frac{d^{3} p}{(2 \pi)^{3}}\left[E_{+}(\mathbf{p})\left(a_{\mathbf{p}}^{\dagger} a_{\mathbf{p}}+\frac{1}{2}\right)+E_{-}(\mathbf{p})\left(b_{\mathbf{p}}^{\dagger} b_{\mathbf{p}}+\frac{1}{2}\right)\right],
$$

where energies are

$$
E_{ \pm}=\sqrt{\omega^{2}(\mathbf{p})+\frac{1}{4}\left[B-\theta \omega^{2}(\mathbf{p})\right]^{2}} \pm \frac{1}{2}\left(B+\theta \omega^{2}(\mathbf{p})\right) .
$$

This shows that the free non commutative complex scalar fields is a theory with two types of particles with different dispersion relation. This asymmetry can be interpreted as a particleantiparticle asymmetry and their phenomenological consequences were explored in $[4,5]$.

A natural question raised by this approach is what happens with other fields as gauge and fermionic fields. Let us discuss gauge fields in next subsection and postpone fermionic fields and its phenomenology to subsequent sections.

\subsection{Non commutative Gauge Fields}

We start the discussion with the $U(1)$ gauge field. As in the previous case, the theory is defined by the standard Hamiltonian [6] (for a previous approach see also[7]).

$$
H=\int d^{3} x\left(\frac{1}{2} \vec{\pi}^{2}+\frac{1}{2} \vec{B}^{2}+A_{0} \vec{\nabla} \cdot \vec{\pi}\right),
$$

plus a set of deformed Poisson bracket structure

$$
\begin{aligned}
& {\left[A_{i}(\vec{x}), A_{j}(\vec{y})\right]_{P B}=0} \\
& {\left[A_{i}(\vec{x}), \pi_{j}(\vec{y})\right]_{P B}=\delta_{i j} \delta(\vec{x}-\vec{y}),} \\
& {\left[\pi_{i}(\vec{x}), \pi_{j}(\vec{y})\right]_{P B}=\theta_{i j} \delta(\vec{x}-\vec{y}),}
\end{aligned}
$$

where $\theta_{i j}$ is the most general antisymmetric three dimensional matrix $\theta_{i j}=\varepsilon_{i j k} \theta_{k}$. Note that this term modifies the infrared sector of the theory due to the dimension of $\theta_{k}$. 
Poisson brackets (2.26) break Lorentz invariance and also the gauge symmetry (GS). In fact, gauge transformation is generated by the Gauss law, which is just the condition that primary constrain preserves in time. The primary constrain is not modified, but its time preservation indeed changes because of extra terms coming from the modified Poisson bracket structure.

In order to study just LIV, preserving gauge symmetry, a modified Gauss law must be introduced. A direct calculation shows that

$$
\chi=\nabla \cdot \pi-\theta \cdot \mathbf{B}
$$

with $\theta \cdot \mathbf{B}=\theta_{i} \varepsilon_{i j k} \partial_{j} A_{k}$ is the modified generator for the gauge symmetry. That is, if $\alpha(\mathbf{x})$ is an arbitrary and real function, then

$$
\begin{aligned}
\delta A_{i}(\mathbf{x}) & =\left[A_{i}(\mathbf{x}), \Delta_{\alpha}\right]_{P B} \\
& =\partial_{i} \alpha(\mathbf{x}), \\
\delta \pi_{i}(\mathbf{x}) & =\left[\pi_{i}(\mathbf{x}), \Delta_{\alpha}\right]_{P B} \\
& =0 .
\end{aligned}
$$

where the gauge transform operator $\Delta_{\alpha}$ is

$$
\Delta_{\alpha}=\int d^{3} y \alpha(\mathbf{y}) \nabla[\pi(\mathbf{y})+\theta \times \mathbf{A}(\mathbf{y})] .
$$

This last relation allows to write the modified Hamiltonian which includes now this new gauge symmetry generator, namely

$$
H=\int d^{3} x\left(\frac{1}{2} \pi^{2}+\frac{1}{2} \mathbf{B}^{2}+A_{0} \nabla[\pi+\theta \times \mathbf{A}]\right) .
$$

The Hamiltonian (2.30) with the Poisson bracket structure (2.26) defines a $U(1)$ gauge field theory which breaks Lorentz symmetry.

This model originates modified Maxwell equations

$$
\begin{aligned}
\dot{A}_{i} & =\pi_{i}-\partial_{i} A_{0}, \\
\dot{\pi}_{i} & =(\pi \times \theta)_{i}-(\nabla \times \mathbf{B})_{i} .
\end{aligned}
$$

First equation is the electric field definition

$$
-E_{i} \equiv \dot{A}_{i}+\partial_{i} A_{0}
$$

which allow to write the remaining equations in the usual form. Including the modified Gauss law they read

$$
\begin{aligned}
\nabla \cdot \mathbf{E} & =-\theta \cdot \mathbf{B}, \\
\nabla \cdot \mathbf{B} & =0, \\
\nabla \times \mathbf{E}+\frac{\partial \mathbf{B}}{\partial t} & =0 . \\
\nabla \times \mathbf{B}-\frac{\partial \mathbf{E}}{\partial t} & =\theta \times \mathbf{E} .
\end{aligned}
$$


In this equation $\theta$ plays the role of a "source" term that can be interpreted as a polarization charge and induced current in a medium, in a similar way to the standard electromagnetic theory. In section IV we will discuss the physical implications of this fact.

Now we would like to discuss other issue, related with the Lagrangian formulation of our approach. From here, the generalization for other gauge groups will be straightforward [8].

The set of equations (2.33) can be obtained from a Lagrangian which is constructed from the Hamiltonian (2.30) in the standard way only if dynamical variables are canonical. Then, we need to find a transformation from variables $\left\{\pi_{i}, A_{j}\right\}$ to variables $\left\{\tilde{\pi}_{i}, \tilde{A}_{j}\right\}$ such that the Poison bracket structure (2.26) maps to the canonical one.

This procedure is completely analog to the change of variables, in previous section, which takes the non commutative phase space variables to a set of standard rising and lowering operators. In the present case, transformations read

$$
\tilde{\pi}_{i}=\pi_{i}+\frac{1}{2}(\boldsymbol{\theta} \times \mathbf{A})_{i}, \quad \tilde{A}_{i}=A_{i} .
$$

and the Lagrangian turn out to be

$$
\begin{aligned}
L & =\int d^{3} x\left(\tilde{\pi}_{i} \dot{A}_{i}-H\right), \\
& \left.=\int d^{3} x\left(\mathbf{E}^{2}-\mathbf{B}^{2}+\frac{1}{2} A_{0} \theta \cdot \mathbf{B}-\frac{1}{2} \mathbf{A} \cdot \boldsymbol{\theta} \times \mathbf{E}\right)\right) .
\end{aligned}
$$

Using the standard definition for $F_{\mu v}$ and $\tilde{F}^{\mu v}=\frac{1}{2} \varepsilon^{\mu \nu \lambda \rho} F_{\lambda \rho},(\mu, v, . .=0,1, \ldots 3)$ one finds that

$$
L=\int\left(-\frac{1}{4} F_{\mu v} F^{\mu v}+\frac{1}{2} \theta_{\mu} \tilde{F}^{\mu v} A_{v}\right) d^{3} x .
$$

This approach can be generalized to others gauge groups. For instance in [8], the $S U(2)$ gauge group was studied and it was shown that the Lagrangian density that generalizes (2.36) is

$$
L=-\frac{1}{2} \operatorname{tr}\left\{F_{\mu v} F^{\mu v}\right\}+2 \theta^{\mu} \varepsilon_{\mu v \rho \sigma} \operatorname{tr}\left(A^{v} F^{\rho \sigma}+\frac{2}{3} g A^{v} A^{\rho} A^{\sigma}\right),
$$

Finally, let us point out that the Chern-Simons term appearing in this formulation is not a perturbative contribution, it appears indeed at the same footing as $F^{2}$ in the $g$ expansion.

We would like now to call the attention on the fact that here we have considered commutator deformations only in the momenta, although we start the discussion with the complex non commutative scalar field where deformations in fields commutators also appears.

It is natural to ask, therefore, what kind of modifications suffers Maxwell theory if we consider a Poisson bracket structure modified as

$$
\left[A_{i}(x), A_{j}(x)\right]=\varepsilon_{i j k} \theta_{k} \delta(x-y),
$$

with $\theta_{k}$ a Lorentz violating vector which plays a role of ultraviolet energy scale. The rest Poisson brackets are canonical.

This theory was considered in [9] and we will not give details on its construction. We would like just to say that, as cases presented before, is possible to restore the gauge symmetry. 
The main phenomenological feature of this approach is that it presents a birefringence effect with polarization planes shifted by an amount proportional to

$$
\omega^{2} \theta \cos \alpha
$$

where $\theta$ is the modulus of $\theta_{k}$ and $\alpha$, the angle between the wave vector $\vec{k}$ and $\theta_{k}$. This frequency dependent behavior is distinctive and it does not appear when noncommutativity in space-time is considered. It is a pure field theory result.

Our last example of non commutative fields is the fermionic case. The next section is devoted to this issue.

\section{Neutrino physics and noncommutative fermionic fields}

A natural question, considering all the previous discussion, is what happens with the fermions fields under a deformation of Poisson structure.

From the phenomenological point of view, our approach is a mechanism that offers an alternative way out to the problem of neutrino oscillations. Let us emphasize that several papers have already dealt with effects of Lorentz [10] and CPT violation [11] in this scenario, however the model we present here has the advantage that depends on a few parameters, as was discussed in previous sections.

Since we are mainly interested in the neutrino sector, and particularly in the problem of oscillations, let us briefly review the situation [12]. Neutrino oscillation is a phenomenological model proposed to explain the deficit of solar and atmospheric neutrinos in fluxes measured on earth [13].

The key idea of this mechanism is to assume that neutrino are massive particles which, upon propagation, oscillates between different flavor eigenstates. In its simplest form, the oscillation between two flavors $i, j$ is considered and it is shown that the probability $P_{i \rightarrow j}(t)$ for specie $i$ to oscillates to $j$ after a time $t$ is

$$
P_{i \rightarrow j}(t)=\sin ^{2}\left(2 \theta_{i j}\right) \sin ^{2}\left(\frac{\left(E_{i}-E_{j}\right) t}{2}\right)
$$

where $\theta_{i j}$ is the mixing angle ${ }^{2}$ and $E_{i(j)}$ is the energy of $i(j)$ species.

It is interesting to note that non vanishing oscillation probability occurs in free space only if there is a non zero $\Delta E_{i j}=E_{i}-E_{j}$ and $\theta_{i j} \neq 0$. Then, if neutrino have equal masses or they are zero, oscillation does not come out.

The standard scenario assumes that neutrino species have small masses and therefore, the probability for oscillation of two neutrino $v_{i}, v_{j}$, in traversing a path length $L$ turns out to be

$$
P_{v_{i} \rightarrow v_{j}}(L)=\sin ^{2}\left(2 \theta_{i j}\right) \sin ^{2}\left(\frac{1.27 \Delta m_{i j}^{2} L}{E}\right),
$$

where $\Delta m_{i j}^{2}=m_{i}^{2}-m_{j}^{2}$ is taken in $(\mathrm{eV})^{2}$, the neutrino energy $E$ in $\mathrm{MeV}$ and $L$ in meters.

\footnotetext{
${ }^{2}$ This angle is introduced to take into account the fact that what propagates is a linear superposition of mass eigenstates.
} 
Clearly, with three families of neutrino there can be only two independent combinations of squared mass differences, lets say $\Delta m_{12}^{2}, \Delta m_{23}^{2}$ from which a solution for solar neutrino as well as atmospheric neutrino puzzles is found. The bounds for this masses in this scenario are [12]

$$
\Delta m_{12}^{2} \leq 10^{-4} \mathrm{eV}^{2}, \quad 10^{-3} \leq \mathrm{eV}^{2} \Delta m_{23}^{2} \leq 10^{-2} \mathrm{eV}^{2} .
$$

From here, the bound for $\Delta m_{13}^{2}$ is fixed.

LSND (Liquid Scintillator Neutrino Detector at Los Alamos) [14] is one of the several experiments that have looked for neutrino oscillations. It has used muon sources from the decay $\pi^{+} \rightarrow \mu^{+}+v_{\mu}$. These muons decay through $\mu^{+} \rightarrow e^{+}+v_{e}+\bar{v}_{\mu}$ and, after a 30 meters long path, the experiment finds the oscillation channel $\bar{v}_{\mu} \rightarrow \bar{v}_{e}$ at $20 \mathrm{MeV} \leq E_{v_{\mu}} \leq 58.2 \mathrm{MeV}$ with probability of $0.26 \%$. According to (3.2), the mass difference involved in this process should be

$$
\Delta m^{2}<1 \mathrm{eV}
$$

Analysis of the MiniBooNE experiment [15] will confirm or discard this result. However, if it is true, we must face a puzzle within the context of three families neutrinos. An explanation of this anomaly, compatible with the standard model, may require the existence of sterile neutrinos [16].

There is a different approach that might solve the puzzle. As was pointed out first by Coleman and Glashow [17] and more extensively developed by Kostelecky and collaborators [18], a departure from Lorentz and/or CPT symmetry in the neutrino sector, gives raise to oscillations as neutrino propagates in free space, even for massless neutrinos.

From (3.1) is clear that the oscillation probability does not vanish if the mixing angle is not zero and the energy of specie 1 is different from specie 2 . This condition satisfies if neutrino masses are different in a theory respecting Lorentz and CPT symmetry (the standard case), but if one of these symmetries is broken, a non zero probability can be obtained.

Note that it is enough to have different dispersion relations for different neutrino species in order to fulfill previous requirements. But this is indeed the case for noncommutative fields approach if we choose non standard commutators among different particle species. In fact, noncommutative scalar field is the first example of this kind, since there field $\phi$ and its conjugate $\phi^{\dagger}$ have a non standard Poisson bracket.

The final result is that, due to non standard anticommutators for massless fermionic fields, dispersion relations are species dependent and a non vanishing oscillation probability is obtained. In what follows we will discuss the technicalities of this approach [19].

In the chiral basis, which is more convenient for our analysis, the Hamiltonian density has the form

$$
\mathscr{H}=i\left(\psi_{L}^{i \dagger} \vec{\sigma} \cdot \vec{\nabla} \psi_{L}^{i}-\psi_{R}^{i \dagger} \vec{\sigma} \cdot \vec{\nabla} \psi_{R}^{i}\right)
$$

where the superscript $i=\{1,2\}$ runs over the flavor quantum number (sum over repeated indexes).

The non-commutative theory is obtained by deforming the canonical anti-commutation relations while maintaining the form of the Hamiltonian density (3.5). We postulate the deformed equal-time anti-commutation relations to have the form (with all others vanishing)

$$
\begin{aligned}
& \left\{\psi_{L}^{i}(\mathbf{x}), \psi_{L}^{j \dagger}(\mathbf{y})\right\}=A^{i j} \delta^{(3)}(\mathbf{x}-\mathbf{y}), \\
& \left\{\psi_{R}^{i}(\mathbf{x}), \psi_{R}^{j \dagger}(\mathbf{y})\right\}=B^{i j} \delta^{(3)}(\mathbf{x}-\mathbf{y}),
\end{aligned}
$$


where $A^{i j}$ and $B^{i j}$ are $2 \times 2$ matrices with constant, complex elements in general, but if we want to maintain rotational invariance, they can be chosen to have the forms

$$
A=\left(\begin{array}{cc}
1 & \alpha \\
\alpha^{*} & 1
\end{array}\right), \quad B=\left(\begin{array}{cc}
1 & \beta \\
\beta^{*} & 1
\end{array}\right),
$$

so that the complex parameters $\alpha, \beta$ can be thought of as the parameters of deformation. Clearly, these deformed anti-commutation relations reduce to the conventional ones when the parameters of deformation vanish.

Equation of motions in the momentum space read

$$
\begin{aligned}
& E \psi_{L}^{i}=-A^{i j}\left(\vec{\sigma} \cdot \vec{p} \psi_{L}^{j}\right), \\
& E \psi_{R}^{i}=B^{i j}\left(\vec{\sigma} \cdot \vec{p} \psi_{R}^{j}\right) .
\end{aligned}
$$

The energy spectrum can be find independently for (3.9) and (3.10). If we take the first one, it is straightforward to find a diagonalization matrix for $A$

$$
D=\frac{1}{\sqrt{2}}\left(\begin{array}{cc}
\frac{|\alpha|}{\alpha} & 1 \\
-\frac{|\alpha|}{\alpha} & 1
\end{array}\right)
$$

and then

$$
\begin{aligned}
& E_{ \pm}^{1}= \pm(1+|\alpha|)|\vec{p}| \\
& E_{ \pm}^{2}= \pm(1-|\alpha|)|\vec{p}|
\end{aligned}
$$

are the eigenvalues in (3.9).

A similar analysis for (3.10) gives

$$
\begin{aligned}
& E_{ \pm}^{1}= \pm(1+|\beta|)|\vec{p}|, \\
& E_{ \pm}^{2}= \pm(1-|\beta|)|\vec{p}| .
\end{aligned}
$$

Since $\psi_{L}^{i}$ does not diagonalize the Hamiltonian -eigenvectors are $D \psi_{L}^{i}$, which are a linear combination of $\psi_{L}^{1}$ and $\psi_{L^{-}}^{2}$ the time evolution of this field gives rise to a linear combination of species 1 and 2, namely a neutrino initially in the state $\psi_{L}^{1}$ would evolve in time as

$$
\begin{aligned}
\psi_{L}^{1}(t) & =\cos \theta_{12} \tilde{\psi}_{L}^{1}(t)-\sin \theta_{12} \tilde{\psi}_{L}^{2}(t) \\
& =\left[\left(\cos ^{2} \theta_{12} e^{-i E_{+}^{1} t}+\sin ^{2} \theta_{12} e^{-i E_{+}^{2} t}\right) \psi_{L}^{1}(0)\right. \\
& \left.+\frac{1}{2} \sin 2 \theta_{12}\left(e^{-i E_{+}^{1} t}-e^{-i E_{+}^{2} t}\right) \psi_{L}^{2}(0)\right] e^{i \vec{p} \cdot \vec{x}} .
\end{aligned}
$$

Therefore, after a path of length $L$, the probability of finding the state $\psi_{L}^{2}$ in the beam is given by

$$
P_{v_{1} \rightarrow v_{2}}=\sin ^{2}\left(2 \theta_{12}\right) \sin ^{2}(|\alpha| E L),
$$

where we have used the fact that for $|\alpha| \ll 1, E \approx|\vec{p}|$. 
Same arguments demonstrate that, for antineutrino propagation, the probability for oscillations is

$$
P_{\bar{v}_{1} \rightarrow \bar{v}_{2}}=\sin ^{2}\left(2 \theta_{12}\right) \sin ^{2}(|\beta| E L) .
$$

The important thing to note here is that

$$
P_{v_{1} \rightarrow v_{2}} \neq P_{\bar{v}_{1} \rightarrow \bar{v}_{2}}
$$

which is a consequence of $C P T$ and Lorentz invariance violation.

Two comments are in order here. First, noncommutative fermionic fields have a mixing angle equals $\pi / 4$, and then is consistent with the so called Large Mixing Angle (LMA) scenario. However, we have introduced in all the discussion a $\theta_{i j}$, in order to mimic that standard case, but it does not appear in a pure noncommutative fermionic theory. Secondly, our description has a linear dependence on the energy, what could be disturbing, but is a natural consequence of the fact that we have incorporated two dimensionless parameters on the dispersion relation.

From previous results we can compute bounds for deformation parameters as follow. Assuming that flavor oscillations involve only pairs of neutrinos, when dealing with three families we must generalize the parameter $\alpha$ to $\alpha_{i j}$ (and the same for antineutrinos). Therefore, flavor oscillation probabilities are

$$
\begin{aligned}
& P_{v_{i} \rightarrow v_{j}}=\sin ^{2}\left(2 \theta_{i j}\right) \sin ^{2}\left(\left|\alpha_{i j}\right| E L\right), \\
& P_{\bar{v}_{i} \rightarrow \bar{v}_{j}}=\sin ^{2}\left(2 \theta_{i j}\right) \sin ^{2}\left(\left|\beta_{i j}\right| E L\right) .
\end{aligned}
$$

From the solar neutrino experiments, oscillations of the flavors $1 \rightarrow 2$ are involved, while the channel $2 \rightarrow 3$ is related with atmospheric neutrino oscillations. Therefore, from (3.17) next bounds on $\alpha$ parameter is found

$$
\begin{aligned}
& \left|\alpha_{12}\right| \leq 10^{-17} \\
& \left|\alpha_{23}\right| \leq 10^{-22}
\end{aligned}
$$

LSND, by other hand, due to the fact that involves antineutrinos, gives a bound for $\beta$ parameter

$$
\left|\beta_{12}\right| \leq 10^{-16}
$$

It is clear that within this scenario, all the experimental results can be naturally explained.

Finally we would like to call the attention on the fact that here neutrino are massless particles and, looking at dispersion relations, we realize that the origin of the energy difference between species can be understood also as a difference in the propagation velocities. Since particles are massless, we would say that particles propagates with different speed of light.

\section{Phenomenological consequences of noncommutative fields in the early universe.}

This section is devoted to analyze possible phenomenological consequences derived from models based on noncommutative fields. 
A consequences of this model which is common for scalars and massless fermions is the asymmetry of the dispersion relation for particles and antiparticles.

For the scalar field, situation is rather simple and the asymmetry can be checked at the level of quantum Hamiltonian (2.23) or at the level dispersion relation (2.24). This theory has two scales, one infrared $B$ and other ultraviolet $\theta$. At momentum $\mathbf{p}$ such that $B<<\omega(\mathbf{p})<<\theta^{-1}$ energy (2.24) satisfy $E_{+} \sim E_{-} \sim \omega(\mathbf{p})$ and we are in the Lorentz invariant region.

Consider a system with this two types of particles in thermodynamical equilibrium at temperature $T$. The density $n / V$ of each specie contained in a volume $V$ (with zero chemical potential) is [5]

$$
\begin{aligned}
& n_{+}=4 \pi \int_{0}^{\infty} \frac{p^{2} d p}{e^{E_{+} / T}-1}, \\
& n_{-}=4 \pi \int_{0}^{\infty} \frac{p^{2} d p}{e^{E_{-} / T}-1} .
\end{aligned}
$$

For a temperature $T$ such that $\theta T<<B / T<<m / T<<1$, there is a tiny asymmetry in the dispersion relation due to the infrared scale and then, a tiny asymmetry in the content of baryonic matter-antimatter content in the volume $V$. In fact

$$
\frac{n_{+}-n_{-}}{n_{-}} \sim \frac{B}{T},
$$

as it is expected if CPT violating effects are tiny.

This example shows that a baryon asymmetry can be generated without departure from thermal equilibrium and it suggests a critical reevaluation of the third criterion of Sakharov for baryogenesis [20].

Since this effect is related only with the asymmetry on the dispersion relation for particles and antiparticles, one could wonder what happens with fermions.

Situation is similar to the previous one. There is an asymmetry due to the different dispersion relations for neutrinos and antineutrinos ${ }^{3}$ and therefore a the ratio of the neutrino density to the antineutrino density, in equilibrium at certain temperature is different from one.

Calculation in this case is more involved due to the presence of different flavors and to the oscillations between them. For two species, however, this problem is formally equivalent to a quantum mechanical two level system with a Hamiltonian which is responsible for the for inducing transition between levels.

The crucial step is to identify this Hamiltonian responsible for the transitions, lets say $v_{e} \rightarrow v_{\mu}$. According to Stodolsky [22] and others [23] this Hamiltonian is

$$
H=\vec{\sigma} \cdot \vec{V},
$$

with $|\vec{V}|=\left|E_{1}-E_{2}\right|$.

Following the results of previous section, we find that the Hamiltonian for a system violating CPT and Lorentz symmetries can be written in this two dimensional space as

$$
\begin{aligned}
H_{+} & =2|\alpha| \vec{\sigma} \cdot \vec{p}, \\
H_{-} & =2|\beta| \vec{\sigma} \cdot \vec{p} .
\end{aligned}
$$

\footnotetext{
${ }^{3}$ In this case, however, the distinction between particles and antiparticles is more subtle, but the quantum Hamiltonian of the theory can also be written as two types of particles with different frequencies[21]
} 
Then, if there is a CPT and Lorentz invariance violation, oscillations neutrino-neutrino and antineutrino-antineutrino take place with different probabilities leading to a neutrino asymmetry.

With present data on neutrino experiments, this asymmetry could be evaluated, however, it seems to premature to carry this analysis at this stage, when still LSND experiment requires a confirmation.

We will finish this section with some comments on the cosmological implications of noncommutative $U(1)$ gauge field. As we mentioned in section II.A, modified Maxwell equations (2.33) mimics standard Maxwell equations with sources, but there is an important difference:electrostatic and magnetostatic appears mixed and then the presence of polarization implies a magnetization and viceversa.

Remarkably, is this structure which offers an alternative to the dynamo mechanism to generate the so called primordial magnetic field. In fact, it was shown in [8] that modified equations admits a solution with the shape

$$
\begin{aligned}
& \mathbf{B}=\mathbf{B}^{(0)}+\mathbf{B}^{(2)}+\mathbf{B}^{(4)}+\ldots \mathbf{B}^{(2 n)}+\ldots, \\
& \mathbf{E}=\mathbf{E}^{(0)}+\mathbf{E}^{(1)}+\mathbf{E}^{(3)}+\ldots \mathbf{B}^{(2 n+1)}+\ldots,
\end{aligned}
$$

where superindices stand for the exponent in $\theta$ in the series expansion. In accordance with experiments, $E$ is always a lower order of magnitude that the magnetic field.

Similarly to a ferromagnetic media, the system might evolve to a stable state with permanent magnetic and/or electric field because the previous expansion not necessarily converges.

Previous mechanism, therefore, is a possible candidate for an alternative explanation to the dynamo mechanism of the primordial magnetic field observed in universe.

\section{Conclusions and outlook}

We have explored phenomenological consequences of Lorentz and CPT symmetries violation through the so called non commutative field theory.

For complex scalar field we have shown how quantum noncommutative field theory can be constructed as a superposition of anisotropic harmonic oscillators. Quantum fermionic fields can be constructed in a similar way and will be reported in a forthcoming paper [21].

The common feature of these approaches is their particle-antiparticle asymmetry manifested in dispersion relations. We have explored the possibility of using this as a possible mechanism to generate baryon-antibaryon asymmetry as well as neutrino anti-neutrino. In the firs case, we have shown how that can be compatible with thermal equilibrium scenario.

For neutrinos, this asymmetry is also the mechanism that allow flavor oscillations and then, two apparently disconnected problems could be explained by the same mechanism. In other words, if CPT and Lorentz are violated, would be possible, in principle, to calculate the excess of neutrinos respect antineutrinos in the universe.

In the gauge field sector, noncommutative fields offers an alternative process to the dynamo mechanism in order to explain interstellar magnetic fields.

In conclusion, a tiny Lorentz symmetry violation open doors to explain observations which can not be accommodate in the conventional physics. Noncommutative fields, by other hand, is a 
description that incorporate this properties in economical way, that is, it depends on a small number of parameters and, just at level of free theory exhibits features that might explain these phenomena.

\section{Acknowledgments}

This work was supported in part by US DOE Grant number DE-FG-02-91ER40685, FONDECYTChile grants 1050114, 1060079 and 2105016 and a FULBRIGHT grant (JL).

\section{References}

[1] For a review see e.g. J. Kogut and K. Wilson, Phys. Rept. 12, 75 (1974).

[2] See for example, C. Itzikson and J. B. Zuber, Quantum Field Theory, (1980)

[3] J.M. Carmona, J.L. Cortes, J. Gamboa and F. Mendez; JHEP 0303, 058 (2003).

[4] J.M. Carmona, J.L. Cortes, J. Gamboa and F. Mendez Phys. Lett. B565, 222 (2003).

[5] J. M. Carmona, J. L. Cortes, A. Das, J. Gamboa and F. Mendez, Mod. Phys. Lett. A21, 883 (2006).

[6] J. Gamboa and J. Lopez-Sarrion, Phys.Rev.D71, 067702 (2005).

[7] S. Carroll, G. B. Field and R. Jackiw, Phys. Rev. D41, 1231 (1990); A. A. Andrianov, P. Giacconi and R. Soldati, JHEP 0202, 030 (2002).

[8] H. Falomir, J. Gamboa, J. Lopez-Sarrion, F. Mendez and A. J. da Silva, Phys. Lett. B632, 740 (2006) and ibid Phys. Rev. D74, 047701 (2006).

[9] J. Gamboa, J. Lopez-Sarrion and A. P. Polychronakos Phys.Lett. B634, 471 (2006).

[10] M. Gasperini, Phys. Rev. D38, 2635; S. L. Glashow, A. Halprin, P. I. Krastev, C. N. Leung and J. Pantaleone, Phys. Rev. D56, 2433 (1997); R. Foot, C. N. Leung and O. Yasuda, Phys. Lett. B443, 185 (1998).

[11] S. Coleman and S. L. Glashow, Phys. Lett. B405, 249 (1997).

[12] For a recent review on neutrino data and experiments, see for example A. Strumia and F. Vissani , hep-ph/0606054 and references therein.

[13] G. L. Fogli, E. Lisi, A. Marrone and G. Scioscia, Phys. Rev. D60, 053006 (1999); B. Pontecorvo, J. Exp. Theor. Phys. (USSR), 34, 247 (1958); L. Wolfenstein, Phys. Rev. D17 , 2369 (1978); S. P. Mikheyev and A. Yu. Smirnov, Sov. J. Nucl. Phys. 42, 913 (1985).

[14] LSND collaboration, C. Athanassopoulos et al., Phys. Rev. Lett. 81, 1774 (2003); LSND collaboration, A. Aguilar et al. Phys. Rev. D64, 112007 (2001).

[15] MiniBooNE collaboration homepage:www-boone.fnal.gov

[16] See S. L. Glashow, Phys. Lett. B256, 255 (1991); J. W. F. Valle and D. Tommasini and J. T. Peltoniemi, Phys. Lett. B298, 383 (1993).

[17] S. Coleman and S. L. Glashow, Phys. Rev. D59, 116008 (1999).

[18] V. A. Kostelecky and M. Mewes in Phys. Rev. D69, 016005 (2004), T. Katori, A. V. Kostelecky and R. Tayloe, hep-ph/0606154.

[19] P. Arias, Ashok Das, J. Gamboa, J. Lopez-Sarrion and F. Mendez, e-Print Archive: hep-ph/0608007 
[20] A. D. Sakharov, JETP Lett 6, 24 (1967).

[21] P. Arias, Ashok Das, J. Gamboa, J. Lopez-Sarrion and F. Mendez, in preparation

[22] L. Stodolsky, Phys. Rev. D36, 2273 (1987); L. Stodolsky, preprint MPI-PAE/PTh 33/88.

[23] V. A. Kostelecky and M. Mewes in Phys. Rev. D69, 016005 (2004), T. Katori, A. V. Kostelecky and R. Tayloe, hep-ph/0606154. 